\title{
Critically ill setting / productivity grid (CISPG): proposal for distribution of productive time of intensivists in a critical care medicine department (CCMD) considering 'outreach'
}

\author{
J Ruiz Moreno ${ }^{1 *}$, JM Nicolás Arfelis², V Gómez Tello ${ }^{3}$, MJ Esteve Paños ${ }^{1}$, R Corcuera Romero de la Devesa ${ }^{1}$, \\ E González Marín ', P Castro Rebollo², P Merino de $\mathrm{Cos}^{4}$, N Franco Garrobo5 ${ }^{5}$, F Baigorri González ${ }^{1}$
}

From ESICM LIVES 2015

Berlin, Germany. 3-7 October 2015

\section{Introduction}

The distribution of intensivists productive time is a classical controversial subject. However it is true that such a professional can practice their profession both within and outside (outreach) the ICU. It is also true also that intensivists serve patients, teaching and research, as well as consider the clinical management $(\mathrm{CM})$ and the risk management (RM).

\section{Objectives}

To propose a CISPG to distribute the productive time of intensivists both in the ICU and outreach.

\section{Methods}

Setting - CCMD A) level III, 14 beds; and CCMD B) level IIb, 10 beds.

Definition of 'productivity': 1. Assistance (1.1. ICU assistance and 1.2. SDU assistance. 2. Teaching. 3. Research. 4. Risk management (RM). 5. Clinical management $(\mathrm{CM})$.

CCMDs features:

CCMD A: 8 ICU beds and 6 SDU beds. ICU assistance, outreach assistance, teaching, experimental and epidemiological research, RM, and CM.

CCMD B: 6 ICU beds and 4 SDU beds. ICU assistance, outreach assistance, teaching, epidemiological research, RM, and CM.

CISPG:

${ }^{1}$ Quirón Salud Hospital Universitario Sagrat Cor, Critical Care Department, Barcelona, Spain

Full list of author information is available at the end of the article
Abscissas (productivity between 08:00 and 17:00): ICU assistance, outreach, teaching, research, RM, and CM.

Ordinates (setting): ICU, SDU, hospital ward (pre and postcritically ill), emergency room (precritically ill and CIP)

3 medical professional job categories: senior, chief clinical (CC) and head of CCMD (HD).

Physician on call are not considered.

Annual working time per physician: 1750 hours

Times adjusted to each specific productivity (sum total $\%=100)$.

It is established $1 \mathrm{HD}, 1 \mathrm{CC}$ for attending CCMDs A and $\mathrm{B}$, and respectively, 4 and 3 seniors.

\section{Results}

\section{Conclusions}

The MAPEC facilitates the distribution of specific work time for intensivists attending both the setting and structured productivity.

\section{Authors' details}

${ }^{1}$ Quirón Salud Hospital Universitario Sagrat Cor, Critical Care Department, Barcelona, Spain. ${ }^{2}$ Hospital Clinic de Barcelona, Critical Care Department, Barcelona, Spain. ${ }^{3}$ Moncloa Hospital Universitario, Critical Care Department, Madrid, Spain. ${ }^{4}$ Hospital Can Misses, Critical Care Department, Ibiza, Spain. ${ }^{5}$ Hospital Universitario Móstoles, Critical Care Department, Móstoles, Spain.

Published: 1 October 2015

\section{References}

1. Weiss M, Marx G, Vagts DA, Schleppers A, Leidinger W, Sehn N, et al: Calculation of personnel requirement "intensive care medicine" 2012 - 
Table 1. CISPG

\begin{tabular}{|c|c|c|c|c|c|c|}
\hline Setting & Hours & Assistance \% & Teaching \% & Research \% & CM \% & RM \% \\
\hline $\begin{array}{l}\text { ICU } \\
\text { Assistance } \\
\text { Teaching } \\
\text { Research CM } \\
\text { RM }\end{array}$ & $\begin{array}{l}\text { ICU - } 8050 \text { Assist - } 3675 \\
\text { Teach - } 875 \text { Resear- } \\
875 \text { CM - } 1225 \text { RM - } \\
1400\end{array}$ & $\begin{array}{l}\text { Senior } 160 \text { Senior } 2 \\
60 \text { Senior } 360 \mathrm{Cl} \text {. } \\
\text { chief } 20 \text { Head Dp } \\
20\end{array}$ & $\begin{array}{l}\text { Senior } 110 \text { Senior } 2 \\
10 \text { Senior } 310 \mathrm{Cl} \text {. } \\
\text { chief } 10 \text { Head Dp } \\
10\end{array}$ & $\begin{array}{l}\text { Senior } 110 \text { Senior } 2 \\
10 \text { Senior } 310 \mathrm{Cl} \text {. } \\
\text { chief } 10 \text { Head Dp } \\
10\end{array}$ & $\begin{array}{l}\text { Senior } 15 \text { Senior } 2 \\
5 \text { Senior } 35 \mathrm{Cl} \text {. } \\
\text { chief } 15 \text { Head Dp } \\
40\end{array}$ & $\begin{array}{l}\text { Senior } 115 \text { Senior } 2 \\
15 \text { Senior } 315 \mathrm{Cl} \text {. } \\
\text { chief } 20 \text { Head Dp } \\
15\end{array}$ \\
\hline $\begin{array}{l}\text { SDU } \\
\text { Assistance } \\
\text { Teaching } \\
\text { Research CM } \\
\text { RM }\end{array}$ & $\begin{array}{l}\text { SDU -1487 Assist -787 } \\
\text { Teach - } 175 \text { Resear- } \\
175 \text { CM - } 87 \text { RM - } 262\end{array}$ & $\begin{array}{l}\text { Senior } 430 \mathrm{Cl} \text {. chief } \\
10 \text { Head Dp } 10\end{array}$ & Senior 410 & Senior 410 & Senior 45 & Senior 415 \\
\hline Hosp. ward & Hosp. ward 525 & $\begin{array}{l}\text { Senior } 415 \mathrm{Cl} \text {. chief } \\
10 \text { Head Dp } 5\end{array}$ & & & & \\
\hline Emergency & Emergency 437 & $\begin{array}{l}\text { Senior } 415 \mathrm{Cl} \text {. chief } \\
10 \text { Head Dp } 5\end{array}$ & & & & \\
\hline Global hours & 10500 & 5425 & 1050 & 1050 & 1312 & 1662 \\
\hline
\end{tabular}

Revision of the 2008 calculation tool for the medical profession.

Anasthesiologie und Intensivmedizin 2012, 53(3):S50-S62.

2. Ruiz J: Asignatura 'Gestión Clínica en Medicina Intensiva', especialidad

'Atención Integral al Enfermo Crítico y Emergencias', Master

Universitario en Investigación Clínica Facultad de Medicina, Universidad de Barcelona. 2015, Edición 2014-2015.

doi:10.1186/2197-425X-3-S1-A481

Cite this article as: Ruiz Moreno et al:: Critically ill setting / productivity grid (CISPG): proposal for distribution of productive time of intensivists in a critical care medicine department (CCMD) considering 'outreach'. Intensive Care Medicine Experimental 2015 3(Suppl 1):A481.

\section{Submit your manuscript to a SpringerOpen ${ }^{\mathcal{O}}$ journal and benefit from:}

- Convenient online submission

- Rigorous peer review

- Immediate publication on acceptance

- Open access: articles freely available online

- High visibility within the field

- Retaining the copyright to your article

Submit your next manuscript at $\gg$ springeropen.com 